T. R. Shemanske

Nagoya Math. J.

Vol. 143 (1996), 147-169

\title{
NEWFORMS OF HALF-INTEGRAL WEIGHT
}

\author{
THOMAS R. SHEMANSKE
}

\section{Background}

Two very different definitions of a newform of half-integral weight are present and continued to be developed in the literature. The first definition originated with Serre and Stark for forms of weight $1 / 2$ [5], and is analogous to the definition of newform for integral weight forms, which uses forms of lower level and shifts of such forms $\left(f(\tau) \mapsto f \mid B_{d}=f(d \tau)\right)$ to characterize the notion of oldforms. The second definition originated with Kohnen for half-integral weight forms of squarefree level [1], who used forms of lower level and their image under the $U_{m^{2}}$ operator $\left(f=\sum a_{n} e^{2 \pi i n \tau} \mapsto f \mid U_{m^{2}}=\sum a_{n m^{2}} e^{2 \pi i n \tau}\right)$ to define the notion of oldforms. The choice of the $U_{m^{2}}$ operator over the shift operator $B_{d}$ seems a propitious one, since the $U$ operator commutes with the action of the Shimura lift, while the shift operator $B$ does not. More to the point, Kohnen was able to develop a newform theory on a distinguished subspace of the full space of cusp forms (now referred to as the Kohnen subspace), and obtained a multiplicity-one result (with respect to Hecke eigenvalues) for half-integral weight newforms in this subspace. Even nicer, the multiplicity-one result was established by showing that there is a one-to-one correspondence between newforms of level $4 N$ in the subspace and the newforms of integral weight of level $N$.

Several others provided further generalizations of the newform theory using both definitions of half-integral weight newforms ([1], [3], [4], [6], [7], [12]). By far the most dramatic development of this theory was obtained recently by Ueda [12]. In this paper, he obtains for arbitrary level a multiplicity-one theorem on a certain subspace of the Kohnen subspace. The generalization from squarefree level to arbitrary level is not at all obvious, and requires very different accommodations for primes dividing the level with multiplicity greater than one. One curious phenomenon which Ueda mentions is that while he was successful in obtaining a multiplicity-one result, newforms of level $4 N$ sometimes correspond (under the Shimura correspondence) to newforms of level a proper divisor of $2 N$. Ueda

Received March 9, 1995. 
continues to refine his subspace in an attempt to remedy this issue.

In joint work with Walling [7], in which we take the alternate definition of newform and work in the full space of cusp forms and not the Kohnen subspace, we determine in two special cases how it is possible for newforms of half-integral weight and squarefree level to correspond to integral weight newforms of any level dividing the expected level given by the Shimura lift. We conjectured that those special cases should generalize.

This paper has two main thrusts. The first is to continue the investigation initiated in [7]. While the desire to obtain multiplicity-one results has directed a great deal of research towards a careful development of the notion of newforms within the Kohnen subspace, a very natural question is to explore whether the multiplicities with which newforms occur on the full space can be determined. In this paper we answer this question for squarefree level. The rather pleasing answer (see Theorem 2.3) is that newforms of half-integral weight correspond to integral weight newforms with multiplicity one or two, and those that occur with multiplicity two can be identified with Kohnen subspaces in such a way that if the full space of newforms (of level $4 N$ ) is decomposed into its Kohnen component and a non-Kohnen component, the newforms in each (corresponding to integral weight newforms of level $N$ or $2 N$ ) occur with multiplicity one.

In the second part of the paper we consider nonsquarefree levels and demonstrate a relation between Ueda's space of newforms and our own (see Theorem 3.1 and Corollary 3.7), by decomposing Ueda's space of newforms into subspaces defined using the alternate characterization of newform. In particular, these results make clear why in certain circumstances, Ueda's newforms often correspond to integral weight newforms whose level is lower than expected. It also brings closer together what were apparently two very different notions of a half-integral weight newform. These results are obtained for both the full space of cusp forms and the Kohnen subspace.

\section{Decomposition theorems-Squarefree level}

Let $N$ be an odd positive integer and $\psi$ an even Dirichlet character defined modulo $4 N$. For an odd integer $k \geq 3$, we denote by $S_{k / 2}(4 N, \phi)$ the space of cusp forms of weight $k / 2$ and character $\phi$ for $\Gamma_{0}(4 N)$. When $k=3$, we take as usual $S_{3 / 2}(4 N, \psi)$ to be the orthogonal complement in the full space of cusp forms of the space generated by theta series attached to quadratic forms in one variable with spherical harmonic (see [12]). For a positive integer $M$, an integer $l \geq 2$, and a Dirichlet character $\varphi$ defined modulo $M$, let $\Im_{l}(M, \varphi)$ denote the space of cusp 
forms of weight $l$ and character $\varphi$ for $\Gamma_{0}(M)$. The rest of the notation and the definition of standard operators like the Hecke operators can be found in [12] or [8].

\subsection{An internal decomposition.}

We recall (see [6]) that the space generated by the oldforms, denoted $S_{k / 2}^{-}(4 N, \phi)$, is defined as

$$
S_{k / 2}^{-}(4 N, \psi)=\sum_{\substack{q|N \\ \operatorname{cond}(\psi)| 4 N / q}} S_{k / 2}(4 N / q, \psi)+\sum_{\substack{q\left|N \\ \operatorname{cond}\left(\psi\left(\frac{q}{*}\right)\right)\right| 4 N / q}} S_{k / 2}\left(4 N / q, \phi\left(\frac{q}{*}\right)\right) \mid B_{q}
$$

where $B_{q}$ is the shift operator taking $f(\tau)$ to $\left(f \mid B_{q}\right)(\tau)=f(q \tau)$. The subspace generated by the newforms, denoted $S_{k / 2}^{+}(4 N, \phi)$ is the orthogonal complement of $S_{k / 2}^{-}(4 N, \psi)$ in the full space $S_{k / 2}(4 N, \psi)$. Our first theorem provides an internal decomposition of the space $S_{k / 2}(4 N, \phi)$ into sums of spaces of newforms when the level $N$ is odd and squarefree.

It turns out to be easier to handle first the case where the character $\psi$ is trivial, and to generalize to nontrivial characters through another means.

THEOREM 2.1. Let $N$ be an odd squarefree integer, and $\psi$ the trivial character modulo $4 N$. Then

$$
S_{k / 2}(4 N, 1)=S_{k / 2}(4 N, \psi)=\bigoplus_{d \mid N} S_{k / 2}^{+}(4 d)
$$

Remark 2.2. It is completely straightforward from the definitions of the spaces of oldforms and newforms to show that $S_{k / 2}(4 N, 1)=\sum_{d \mid N} S_{k / 2}^{+}(4 d)$. Moreover, if these were spaces of integral weight newforms, such a sum would obviously be direct (in fact orthogonal) since newforms of distinct levels have infinitely many distinct eigenvalues under the Hecke operators. Unfortunately for half-integral weight forms, this is very far from true. In fact to describe this phenomenon more clearly is a major focus of this paper. The special cases where $N$ is a prime or the product of two distinct primes were established in [7].

Proof. For notational ease within this proof, we suppress the notation for the weight and character, and write $S(4 d)$ for $S_{k / 2}(4 d, 1)$ and $S^{ \pm}(4 d)$ for $S_{k / 2}^{ \pm}(4 d, 1)$. Write $N=p_{1} p_{2} \cdots p_{r}$, the product of distinct odd primes. We always have

$$
S(4 N)=S^{+}(4 N) \oplus S^{-}(4 N),
$$

and since $\phi=1$, 


$$
S^{-}(4 N)=\sum_{i=1}^{r} S\left(4 p_{1} \cdots p_{r} / p_{i}\right)
$$

Thus we need to show that

$$
S^{-}(4 N)=\bigoplus_{\substack{d \mid N \\ d<N}} S_{k / 2}^{+}(4 d)
$$

As we remarked above, a trivial induction shows that

$$
S^{-}(4 N)=\sum_{\substack{d \mid N \\ d<N}} S_{k / 2}^{+}(4 d) .
$$

We rewrite this sum as

$$
\sum_{\substack{k=0 \\ r-1}} \sum_{\substack{0 \leq \nu_{i} \leq 1 \\ \Sigma \nu_{i}=k}} S^{+}\left(4 p_{1}^{\nu_{1}} p_{2}^{\nu_{2}} \cdots p_{r}^{\nu_{r}}\right) .
$$

We first show that for fixed $k$, the inner sum is direct. There are two cases.

Case 1. For $k=0$, there is only one summand, so the result is clear. When $k=1, r \geq 2$ and the sum becomes $\sum_{i=1}^{r} S^{+}\left(4 p_{i}\right)$. Suppose that $\sum f_{t}=0$ with $f_{i} \in S^{+}\left(4 p_{\imath}\right)$. Then we may assume there exists an index $i$ so that $f_{i}$ is not zero. Then

$$
f_{i}=-\sum_{j \neq \imath} f_{j} \in S^{+}\left(4 p_{\imath}\right) \cap S\left(4 p_{1} \cdots p_{r} / p_{\imath}\right) \subset S(4) \subset S^{-}\left(4 p_{\imath}\right),
$$

which implies $f_{i}=0$, a contradiction.

Case 2. In the remaining case we have $2 \leq k<r$. This time we employ the trace operator (adjoint to the inclusion) to achieve our goal. For properties of the trace operator, see [6] or [5].

Suppose that $\sum_{\sum \nu_{i}=k} S^{+}\left(4 p_{1}^{\nu_{1}} \cdots p_{r}^{\nu_{r}}\right)$ is not direct. Then there exists a nontrivial dependence relation of the form $\sum f_{j_{1} \cdots j_{k}}=0$ with $f_{j_{1} \cdots j_{k}} \in S^{+}\left(4 p_{j_{1}} \cdots p_{j_{k}}\right)$. Fix an index $i_{1} \cdots i_{k}$ for which $f_{i_{1} \cdots i_{k}} \neq 0$, and put $\operatorname{Tr}=\operatorname{Tr}_{4 p_{i_{1}} \cdots p_{i_{k}}}^{4 N}$. We shall show that $\sum f_{j_{1} \cdots j_{k}} \mid \operatorname{Tr}=f_{i_{1} \cdots l_{k}}=0$, providing our contradiction.

Certainly

$$
f_{i_{1} \cdots i_{k}}\left|\operatorname{Tr}=f_{i_{1} \cdots i_{k}}\right| \operatorname{Tr}_{4 p_{i_{1}} \cdots p_{i_{k}}}^{4 N}=f_{i_{1} \cdots i_{k}}
$$

since $f_{i_{1} \cdots i_{k}}$ has level $4 p_{i_{1}} \cdot \cdot p_{\imath_{k}}$. Now consider $f_{j_{1} \cdots j_{k}} \neq f_{i_{1} \cdots i_{k}}$. Let $D=$ $\operatorname{gcd}\left(p_{i_{1}} \cdots p_{i_{k}}, p_{\jmath_{1}} \cdots p_{\jmath_{k}}\right)$, and write $p_{i_{1}} \cdots p_{i_{k}}=D I$ and $p_{j_{1}} \cdots p_{j_{k}}=D J$. Note 
that $I$ and $J$ are both greater than 1 , since $\left\{i_{1}, \ldots, i_{k}\right\} \neq\left\{j_{1}, \ldots, j_{k}\right\}$. Finally, write $N=p_{1} \cdots p_{r}=D I J K$ for some integer $K$.

Then

$$
\begin{aligned}
f_{j_{1} \cdots j_{k}} \mid \operatorname{Tr}_{4 p_{i_{1}} \cdots p_{i_{k}}}^{4 N} & =f_{j_{1} \cdots j_{k}} \mid \operatorname{Tr}_{4 D I}^{4 D I J K} \\
& =f_{j_{1} \cdots j_{k}} \mid \operatorname{Tr}_{4 D}^{4 D J K} \text { since } \operatorname{gcd}(I, J K)=1
\end{aligned}
$$

and letting $p$ be any prime dividing $J$ (recall $J>1$ )

$$
\begin{aligned}
& =f_{j_{1} \cdots j_{k}}\left|\operatorname{Tr}_{4 D J}^{4 D J K}\right| \operatorname{Tr}_{4 D J / p}^{4 D J} \mid \operatorname{Tr}_{4 D}^{4 D J / p} \\
& =f_{j_{1} \cdots j_{k}}\left|\operatorname{Tr}_{4 D J / p}^{4 D J}\right| \operatorname{Tr}_{4 D / p}^{4 D J} \text { since } f_{f_{1} \cdots j_{k}} \text { has level } 4 D J \\
& =0 \mid \operatorname{Tr}_{4 D}^{4 D J / p}=0 \text { by Theorem } 5.2 \text { of }[6] .
\end{aligned}
$$

So we now have that

$$
S^{-}(4 N)=\sum_{k=0}^{r-1} \bigoplus_{\substack{0 \leq \nu_{i} \leq 1 \\ \Sigma \nu_{i}=k}} S^{+}\left(4 p_{1}^{\nu_{1}} p_{2}^{\nu_{2}} \cdots p_{r}^{\nu_{r}}\right)
$$

We now show that the outer sum is direct. Suppose not. Then there exists a nontrivial dependence relation

$$
f_{0}+f_{1}+\cdots+f_{r-1}=0 \text { where } f_{k} \in \underset{\substack{0 \leq \nu_{i} \leq 1 \\ \Sigma \nu_{i}=k}}{\bigoplus} S^{+}\left(4 p_{1}^{\nu_{1}} p_{2}^{\nu_{2}} \cdots p_{r}^{\nu_{r}}\right) \text {. }
$$

Let $\nu^{*}$ be the largest index so that $f_{\nu^{*}} \neq 0$, and write

$$
f_{\nu^{*}}=\sum g_{i_{1} \cdots i_{\nu^{*}}} \text { where } g_{i_{1} \cdots i_{\nu} *} \in S^{+}\left(4 p_{i_{1}} \cdots p_{i_{\nu^{*}}}\right) \text {. }
$$

Choose any index $k_{1} \cdots k_{\nu^{*}}$ for which $g_{k_{1} \cdots k_{\nu^{*}}} \neq 0$, and consider the trace operator $\operatorname{Tr}=\operatorname{Tr}_{4 p_{k_{1}} \cdots p_{k_{\nu} *}}^{4 N}$. Exactly as in the first part of the proof, we see that

$$
f_{\nu^{*}}\left|\operatorname{Tr}=\sum g_{i_{1} \ldots i_{\nu *}}\right| \operatorname{Tr}_{4 p_{k_{1}} \cdots p_{k_{\nu} *}}^{4 N}=g_{k_{1} \cdots k_{\nu} *} .
$$

Now we claim that for $\nu<\nu^{*}, f_{\nu} \mid \operatorname{Tr} \in S^{-}\left(4 p_{k_{1}} \cdots p_{k_{\nu *}}\right)$. This will imply that

$$
f_{\nu^{*}}\left|\operatorname{Tr}=g_{k_{1} \cdots k_{\nu *}}=-\left(f_{1}+\cdots+f_{\nu^{*}-1}\right)\right| \operatorname{Tr} \in S^{-}\left(4 p_{k_{1}} \cdots p_{k_{\nu} *}\right),
$$

forcing $g_{k_{1} \cdots k_{\nu *}}=0$, a contradiction.

To establish the claim, we first note that essentially by definition of the trace operator, $f_{\nu} \mid \operatorname{Tr} \in S\left(4 p_{k_{1}} \cdots p_{k_{\nu} *}\right)$. For $\nu<\nu^{*}$, write

$$
f_{\nu}=\sum g_{l_{1} \cdots l_{\nu}} \text { where } g_{l_{1} \cdots l_{\nu}} \in S^{+}\left(4 p_{l_{1}} \cdots p_{l_{\nu}}\right) \text {. }
$$

Let $D=\operatorname{gcd}\left(p_{l_{1}} \cdots p_{l_{\nu}}, p_{k_{1}} \cdots p_{k_{\nu} *}\right)$, and write $p_{k_{1}} \cdots p_{k_{\nu} *}=D K$ and $p_{l_{1}} \cdots p_{l_{\nu}}$ 
$=D L$ for integers $K$ and $L$. Note that $K>1$ since $\nu<\nu^{*}$, but $L$ could equal 1 . Finally write $N=p_{1} \cdots p_{r}=D K L M$ for some integer $M$, and note that $\operatorname{gcd}(K$, $L M)=1$.

There are two cases. If $L=1$, then $D=p_{l_{1}} \cdots p_{l_{\nu}}$ is a proper divisor of $p_{k_{1}} \cdots p_{k_{\nu} *}$ This implies that $g_{l_{1} \cdots l_{\nu}} \mid \operatorname{Tr}=g_{l_{1} \cdots l_{\nu}}$ since $g_{l_{1} \cdots l_{\nu}}$ has level dividing $p_{k_{1}} \cdots p_{k_{\nu} *}$ Moreover, since $D$ is a proper divisor of $p_{k_{1}} \cdots p_{k_{\nu} *}, g_{l_{1} \cdots l_{\nu}} \in$ $S^{-}\left(4 p_{k_{1}} \cdots p_{k_{2 *}}\right)$.

If $L>1$, then let $p$ be any prime dividing $L$. Then

$$
\begin{aligned}
g_{l_{1} \cdots l_{\nu}} \mid \operatorname{Tr} & =g_{l_{1} \cdots l_{\nu}} \mid \operatorname{Tr}_{4 D K}^{4 D L K M} \\
& =g_{l_{1} \cdots l_{\nu}} \mid \operatorname{Tr}_{4 D}^{4 D L M} \text { since } \operatorname{gcd}(K, L M)=1 \\
& =g_{l_{1} \cdots l_{\nu}}\left|\operatorname{Tr}_{4 D L}^{4 D L M}\right| \operatorname{Tr}_{4 D L / p}^{4 D L} \mid \operatorname{Tr}_{4 D}^{4 D L / p} \\
& =g_{l_{1} \cdots l_{\nu}}\left|\operatorname{Tr}_{4 D L / p}^{4 D L}\right| \operatorname{Tr}_{4 D}^{4 D L / p} \text { since the level of } g_{l_{1} \cdots l_{\nu}} \text { is } 4 D L \\
& =0 \mid \operatorname{Tr}_{4 D}^{4 D L / p} \text { by Theorem } 5.2 \text { of }[6] .
\end{aligned}
$$

Thus $f_{\nu} \mid \operatorname{Tr} \in S^{-}\left(4 p_{k_{1}} \cdots p_{k_{\nu *}}\right)$ which completes the proof the claim, and of the theorem.

\subsection{An external decomposition}

Next we give a correspondence between newforms of half-integral weight and newforms of integral weight. For squarefree level, we shall see that half-integral weight newforms occur with multiplicity 1 or 2 , and those occurring with multiplicity 2 are precisely those with at least one member in the Kohnen subspace of newforms.

Let $N$ be an odd positive squarefree integer, and $k \geq 3$ an odd integer. Ueda $[10, \S 3]$ defines an abstract Hecke algebra $H(N)$ and two representations of it, one in the space of integral weight cusp forms, the other in the space of half-integral weight cusp forms. Isomorphisms (as $H(N)$-modules) between spaces of cusp forms of integral and half-integral weight have the property that an eigenform for a half-integral weight Hecke operator $\tilde{T}_{k / 2}\left(n^{2}\right) \quad($ with $(n, 2 N)=1$ ) corresponds to an eigenform for the integral weight Hecke operator $T_{k-1}(n)$ which has the same eigenvalue as its half-integral weight counterpart. Since the Hecke eigenvalues for $T_{k-1}(p)$ for almost all primes $p$ characterize integral weight newforms precisely, we obtain our multiplicity results as a consequence.

In particular, we have the

THEOREM 2.3. Let $N$ be an odd positive squarefree integer, and $k \geq 3$ an odd integer. Then 


$$
S_{k / 2}^{+}(4 N, 1) \cong \bigoplus_{d \mid N}\left[\Im_{k-1}^{+}(2 d) \oplus 2 \mathfrak{S}_{k-1}^{+}(d)\right]
$$

where the isomorphism is as modules for the Hecke algebra $H(N)$.

Remark 2.4. Note that this theorem says that newforms of half-integral weight, "squarefree level" $4 N$ and trivial character correspond to integral weight newforms of all possible levels dividing $2 N, 2 N$ being the level to which they correspond under any Shimura lift. It is also remarkable to note that viewed in the opposite direction, the theorem says that given any newform $F$ of squarefree level $d$ and trivial character, there exist half-integral weight newforms of level $2 M d$ or $4 M d$ having the same eigenvalues as $F$ for any squarefree integer $M$ prime to $2 d$. Note that while there obviously exist cusp forms of arbitrarily high levels with the same eigenvalues as $F$, it is surprising that newforms should exist with this behavior. In particular, such newforms cannot arise as character twists of half-integral weight newforms of level $4 d$. Moreover, we note that all newforms in $S_{k / 2}^{+}(4 N, 1)$ which correspond to integral weight newforms of level $2 d$ for any $d \mid N$, do in fact occur with multiplicity one.

Before proving the theorem, we state a corollary which connects the notion of newform developed by Kohnen with the one used in this paper which parallels the one for integral weight forms. With the notation as above, denote the Kohnen subspace by $S_{k / 2}(4 N, 1)_{K}$ and the corresponding subspace of newforms (see [1] for definitions) by $S_{k / 2}^{\text {new }}(4 N, 1)_{K}$. By Theorem 2.4 of [7], we know that $S_{k / 2}^{\text {new }}(4 N, 1)_{K}$ $\subset S_{k / 2}^{+}(4 N, 1)$. Let $S_{k / 2}^{\text {new }}(4 N, 1)_{N K}(N K$ meaning nonKohnen) be the orthogonal complement of $S_{k / 2}^{\text {new }}(4 N, 1)_{K}$ in $S_{k / 2}^{+}(4 N, 1)$. Then both $S_{k / 2}^{\text {new }}(4 N, 1)_{K}$ and $S_{k / 2}^{\text {new }}(4 N, 1)_{N K}$ are Hecke (i.e., $\left.H(N)\right)$ submodules of $S_{k / 2}^{+}(4 N, 1)$. From Kohnen's work [1], we know that as $H(N)$-modules $S_{k / 2}^{\text {new }}(4 d, 1)_{K} \cong \mathfrak{S}_{k-1}^{+}(d, 1)$ for any divisor $d$ of $N$. In particular, we obtain the

COROllary 2.5. With the notation as above,

$$
\begin{aligned}
S_{k / 2}^{+}(4 N, 1) & =S_{k / 2}^{\text {new }}(4 N, 1)_{K} \oplus S_{k / 2}^{\text {new }}(4 N, 1)_{N K} \\
& \cong \bigoplus_{d \mid 2 N} \Im_{k-1}^{+}(d) \oplus \bigoplus_{e \mid N} \Im_{k-1}^{+}(e) \\
& \cong \bigoplus_{d \mid 2 N} \Im_{k-1}^{+}(d) \oplus \bigoplus_{e \mid N} S_{k / 2}^{\text {new }}(4 e, 1)_{K} .
\end{aligned}
$$

In particular,

$$
S_{k / 2}^{\text {new }}(4 N, 1)_{N K} \cong \Im_{k-1}^{+}(2 N) \oplus \mathfrak{\Im}_{k-1}^{+}(N) \oplus \sum_{\substack{d ! N \\ d<N}}\left[\Im_{k-1}^{+}(2 d) \oplus 2 \Im_{k-1}^{+}(d)\right] .
$$


Proof (of Theorem 2.3). Once again for convenience we shall abbreviate $S_{k / 2}^{ \pm}(4 N, 1)$ to $S^{ \pm}(4 N)$, and we shall also abbreviate $\Im_{k-1}^{ \pm}(M, 1)$ to $\Im^{ \pm}(M)$. The basic idea of the proof is quite straightforward. First, by the corollary in $\S 3$ of [10], we obtain

$$
S_{k / 2}(4 N, 1)=S_{k / 2}^{+}(4 N, 1) \oplus S_{k / 2}^{-}(4 N) \cong \varsigma_{k-1}(2 N, 1) .
$$

Then we use the integral weight newform theory to decompose $\widetilde{S}_{k-1}(2 N, 1)$ and induction with Theorem 2.1 above to identify the oldform parts. Finally, using semisimplicity of the Hecke algebra, we cancel factors yielding the result.

Write $N=p_{1} \cdots p_{r}$ where the $p_{i}$ are distinct odd primes. Let $\sigma_{0}(n)$ denote the number of positive divisors of $n$. Then as modules for the Hecke algebra $H(N)$, integral weight newform theory yields

$$
\begin{aligned}
& \Im\left(2 p_{1} \cdots p_{r}\right) \cong \underset{d \mid 2 p_{1} \cdots p_{r}}{\bigoplus} \sigma_{0}\left(\frac{2 p_{1} \cdots p_{r}}{d}\right) \Im^{+}(d) \\
& \cong \underset{d i p_{1} \cdots p_{r}}{\bigoplus}\left[\sigma_{0}\left(\frac{p_{1} \cdots p_{r}}{d}\right) \Im^{+}(2 d) \oplus \sigma_{0}\left(\frac{2 p_{1} \cdots p_{r}}{d}\right) \Im^{+}(d)\right] \\
& \cong \underset{d \mid p_{1} \cdots p_{r}}{\bigoplus} \sigma_{0}\left(\frac{p_{1} \cdots p_{r}}{d}\right)\left[\Im^{+}(2 d) \oplus 2 \Im^{+}(d)\right] \\
& \cong \bigoplus_{k=0}^{r} \bigoplus_{\substack{0 \leq \nu_{i} \leq 1 \\
\Sigma \nu_{i}=k}} 2^{r-k}\left[\mathfrak{S}^{+}\left(2 p_{1}^{\nu_{1}} \cdots p_{r}^{\nu_{r}}\right) \oplus 2 \mathfrak{S}^{+}\left(p_{1}^{\nu_{1}} \cdots p_{r}^{\nu_{r}}\right)\right] .
\end{aligned}
$$

On the other hand, by Theorem 2.1, we have

$$
\begin{aligned}
& S\left(4 p_{1}^{\nu_{1}} \cdots p_{r}^{\nu_{r}}\right)=\bigoplus_{d \mid p_{1}^{\nu_{1} \cdots p_{r}^{\nu_{r}}}} S^{+}(4 d)=\bigoplus_{l=0}^{r} \underset{\substack{0 \leq \mu_{i} \leq 1 \\
\Sigma \mu_{i}=l}}{\bigoplus} S^{+}\left(4 p_{1}^{\mu_{1}} \cdots p_{r}^{\mu_{r}}\right)
\end{aligned}
$$

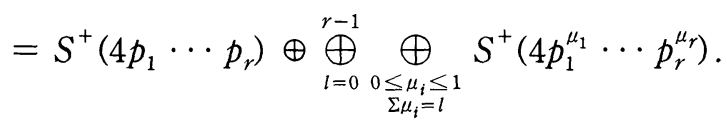

Now we proceed by induction on $r$. The base case $r=0$ being trivial, we assume that $r \geq 1$ and

$$
S^{+}\left(4 p_{1}^{\mu_{1}} \cdots p_{r}^{\mu_{r}}\right) \cong \bigoplus_{d \mid p_{1}^{\mu_{1} \cdots p_{r}^{\mu_{r}}}}\left[\Im^{+}(2 d) \oplus 2 \Im^{+}(d)\right]
$$

for any set of primes with $0 \leq \mu_{i} \leq 1$ and $\sum \mu_{i}=l<r$. We rewrite the above as

$$
S^{+}\left(4 p_{1}^{\mu_{1}} \cdots p_{r}^{\mu_{r}}\right) \cong \bigoplus_{j=0}^{l} \bigoplus_{\substack{0 \leq m_{i} \leq \mu_{i} \\ \Sigma m_{i}=1}}\left[\Im^{+}\left(2 p_{1}^{m_{1}} \cdots p_{r}^{m_{r}}\right) \oplus 2 \Im^{+}\left(p_{1}^{m_{1}} \cdots p_{r}^{m_{r}}\right)\right]
$$


Combining the above with equations (2.4) and (2.5), we obtain

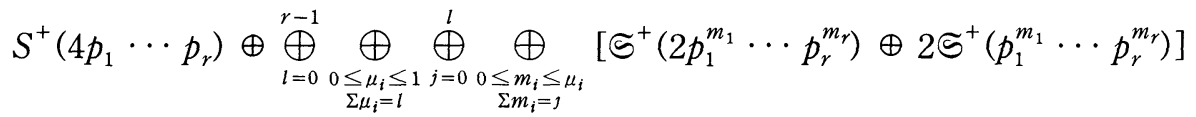

$$
\begin{aligned}
& \cong \bigoplus_{k=0}^{r} \bigoplus_{\substack{\leq \leq \nu_{i} \leq 1 \\
\Sigma \nu_{i}=k}} 2^{r-k}\left[\varsigma^{+}\left(2 p_{1}^{\nu_{1}} \cdots p_{r}^{\nu_{r}}\right) \oplus 2 \Im^{+}\left(p_{1}^{\nu_{1}} \cdots p_{r}^{\nu_{r}}\right)\right]
\end{aligned}
$$

Fix a sequence $\nu_{1}, \ldots, \nu_{r}$ with $0 \leq \nu_{i} \leq 1$ and $\sum \nu_{i}=k<r$. Note that the summand $\left[\Im^{+}\left(2 p_{1}^{\nu_{1}} \cdots p_{r}^{\nu_{r}}\right) \oplus 2 \varsigma^{+}\left(p_{1}^{\nu_{1}} \cdots p_{r}^{\nu_{r}}\right)\right]$ occurs with multiplicity $2^{r-k}$ on the RHS of equation (2.6). We shall show that it occurs with multiplicity $2^{r-k}-1$ on the LHS. Then using semisimplicity of the Hecke algebra, we cancel like terms on both sides of (2.6) to obtain

$$
\begin{aligned}
S^{+}\left(4 p_{1} \cdots p_{r}\right) & \cong \bigoplus_{k=0}^{r} \bigoplus_{\substack{0 \leq \nu_{i} \leq 1 \\
\Sigma \nu_{i}=k}}\left[\mathfrak{S}^{+}\left(2 p_{1}^{\nu_{1}} \cdots p_{r}^{\nu_{r}}\right) \oplus 2 \mathfrak{S}^{+}\left(p_{1}^{\nu_{1}} \cdots p_{r}^{\nu_{r}}\right)\right] \\
& =\bigoplus_{d \mid N}\left[\mathfrak{S}^{+}(2 d) \oplus 2 \mathfrak{S}^{+}(d)\right]
\end{aligned}
$$

as required. We need only establish the claim concerning the multiplicity.

To make an accurate count, we need only count the number of times the $r$-tuple $\left(m_{1}, \ldots, m_{r}\right)=\left(\nu_{1}, \ldots, \nu_{r}\right)$ occurs on the LHS. In the notation of (2.6), we make two simple observations: For $1 \leq i \leq r$, we have $\nu_{i}=m_{i} \leq \mu_{\imath}$ and $j=$ $k=\sum_{i=1}^{r} \nu_{i}=\sum_{i=1}^{r} m_{i} \leq \sum_{i=1}^{r} \nu_{\imath}=l$. Also recall that all entries in the $r$-tuples are zeros or ones. We need to count the number of $r$-tuples $\mu=\left(\mu_{1}, \ldots, \mu_{r}\right)$ which can give rise to the $r$-tuple $\mathbf{m}=\left(m_{1}, \ldots, m_{r}\right)$ subject to the above constraints. We count as a function of $l$.

From the inequality above, we see that $l \geq k$. If $l=k, k=\sum_{i=1}^{r} m_{i}=\sum_{l=1}^{r}$ $\nu_{i}=l$ which says there is only one possible $r$-tuple $\mu$ which admits $\mathbf{m}$. For $l=k$ +1 , we know that any admissible $\mu$ has ones everywhere that $\mathbf{m}$ does, plus exactly one more. Since $k=\sum_{l=1}^{r} m_{\imath}$, there are $n-k$ possible indices for the extra one, and hence $n-k$ possible $r$-tuples $\mu$ which admit $\mathbf{m}$. In general, when $l=k$ $+s(0 \leq s \leq n-k-1)$, any admissible $\mu$ has $s$ additional ones where $\mathbf{m}$ has zeros, and there are $n-k$ indices from which to choose where to place them. Of course, this means that there are $\left(\begin{array}{c}n-k \\ s\end{array}\right) r$-tuples $\mu$ which admit $\mathbf{m}$. Thus in all there are

$$
1+(n-k)+\left(\begin{array}{c}
n-k \\
2
\end{array}\right)+\cdots+\left(\begin{array}{c}
n-k \\
n-k-1
\end{array}\right)=\sum_{s=0}^{n-k-1}\left(\begin{array}{c}
n-k \\
s
\end{array}\right)
$$


admissible $\mu$. Since it is well known that

$$
\sum_{s=0}^{m}\left(\begin{array}{l}
m \\
S
\end{array}\right)=(1+1)^{m}=2^{m}
$$

the number of admissible $\mu$ is clearly $2^{n-k}-1$, as claimed.

\subsection{An extension to quadratic character}

The purpose of this section is to deduce an analog of Theorem 2.3 which holds when the character on the space of half-integral weight forms is an arbitrary quadratic character.

To describe the situation further, we set some notation. As above, let $N$ be an odd positive squarefree integer, and $k \geq 3$ an odd integer. Let $\phi$ be an even quadratic Dirichlet character defined modulo $4 N$. Rather than attempt to derive another internal decomposition for $S_{k / 2}(4 N, \phi)$ as in Theorem 2.1, we shall show that $S_{k / 2}^{+}(4 N, \psi)$ and $S_{k / 2}^{+}(4 N, 1)$ are isomorphic as modules for the Hecke algebra $H(N)$, from which we shall derive the

THEOREm 2.6. Let $N$ be an odd positive squarefree integer, and $k \geq 3$ an odd integer. Let $\phi$ be an even quadratic Dirichlet character defined modulo $4 N$. Then

$$
S_{k / 2}^{+}(4 N, \phi) \cong \bigoplus_{d \mid N}\left[\Im_{k-1}^{+}(2 d) \oplus 2 \Im_{k-1}^{+}(d)\right]
$$

where the isomorphism is as modules for the Hecke algebra $H(N)$.

The idea behind the desired isomorphism is simple. Since $N$ is squarefree, the character $\phi$ is equal to $\left(\frac{Q}{*}\right)$ for some divisor $Q$ of $N$ with (necessarily, since $N$ is squarefree) $\operatorname{gcd}(Q, 4 N / Q)=1$. A generalized Atkin-Lehner involution $W_{Q}$ will act as a "hermitian involution" which commutes with the action of the Hecke operators, and hence provides an isomorphism (as modules for the Hecke algebra) between $S_{k / 2}^{+}(4 N, 1)$ and $S_{k / 2}^{+}\left(4 N,\left(\frac{Q}{*}\right)\right)$.

While $W_{Q}$ operators have been defined in [1], [7], and [12], none of these operators is sufficiently general to give a commutation relation with the shift operators $\left(B_{d}\right)$ which are fundamental to our definition of the space of oldforms (and hence of newforms). So we introduce a slightly more general operator with the desired properties. The desired properties (commutation relations with various other operators, hermitian properties, etc.) are completely analogous to properties for the in- 
tegral weight analog. The proofs are more tedious because of the fussiness of automorphy factors, although there are no significant surprises. Therefore we state the properties and give only the barest of outlines of the proofs.

In defining the $W_{Q}$ operator, there is no reason to assume that $N$ is squarefree or that the character is quadratic. In fact it is quite revealing not to do so, since we shall see why the situation of quadratic character is special.

Let $N$ be a positive integer, and $Q$ an odd divisor of $N$ with $\operatorname{gcd}(Q, 4 N / Q)$ $=1$. Let $\phi$ be an even Dirichlet character defined modulo $4 N$, and let

$$
\gamma_{Q}=\left(\begin{array}{cc}
x & y \\
\frac{4 N}{Q} z & Q w
\end{array}\right) \in \Gamma_{0}\left(\frac{4 N}{Q}\right), \quad \delta_{Q}=\left(\begin{array}{cc}
Q & 0 \\
0 & 1
\end{array}\right), W_{Q}=\gamma_{Q} \delta_{Q} .
$$

For $f \in S_{k / 2}(4 N, \phi)$ define $\tilde{W}_{Q}$ by

$$
f\left|\tilde{W}_{Q}=f\right| \tilde{W}_{Q}(\phi)=\phi_{Q}(y) \phi_{\frac{4 N}{Q}}(x) f \mid \gamma_{Q}^{*} \tilde{\delta}_{Q}
$$

where $\gamma_{Q}^{*}=\left\{\gamma_{Q}, j\left(\gamma_{Q}, z\right)\right\}$ and $\tilde{\delta}_{q}=\left\{\delta_{Q}, Q^{-1 / 4}\right\}$ are elements in the metaplectic cover $\mathscr{G}$ of $G L_{2}^{+}(\mathbf{Q})$ (see Shimura [8]), and $\psi_{Q}$ and $\psi_{\frac{4 N}{Q}}$ are the $Q$ th and $\frac{4 N}{Q}$ th parts of $\phi$. Here for $\gamma \in \Gamma_{0}(4), j(\gamma, z)$ is the standard automorphy factor

$$
j(\gamma, z)=\frac{\theta(\gamma(z))}{\theta(z)}, \quad \theta(z)=\sum_{n \in \mathbf{Z}} e^{2 \pi i n^{2} z} .
$$

It is trivial to check that $\tilde{W}_{Q}$ is well-defined and that Ueda's $\tilde{W}_{Q}$ is a special case of this operator.

Proposition 2.7. The operator $\tilde{W}_{Q}$ maps $S_{k / 2}(4 N, \phi)$ to $S_{k / 2}\left(4 N, \bar{\phi}_{Q} \psi_{\frac{4 N}{Q}}\left(\frac{Q}{*}\right)\right)$.

This follows easily from the observation that for $\gamma \in \Gamma_{0}(4 N)$,

$$
\tilde{W}_{Q}(\phi) \gamma^{*} \tilde{W}_{Q}(\phi)^{-1}=\left(W_{Q} \gamma W_{Q}^{-1}\right)^{*}\left\{1,\left(\frac{Q}{d}\right)\right\}
$$

Next we have the

Proposition 2.8. Let $f \in S_{k / 2}(4 N, \phi)$ then

1. $f\left|\tilde{W}_{Q}(\phi)\right| \tilde{W}_{Q}\left(\bar{\phi}_{Q} \psi_{\frac{4 N}{Q}}\left(\frac{Q}{*}\right)\right)=\left(\frac{-1}{Q}\right)^{k / 2} \bar{\phi}_{\frac{4 N}{Q}}(Q) \phi_{Q}(-1) f$.

2. If $Q^{\prime} \mid 4 N$ with $\operatorname{gcd}\left(Q, \frac{4 N}{Q}\right)=\operatorname{gcd}\left(Q^{\prime}, \frac{4 N}{Q^{\prime}}\right)=\operatorname{gcd}\left(Q, Q^{\prime}\right)=1$, then 


$$
f\left|\tilde{W}_{Q}(\psi)\right| \tilde{W}_{Q^{\prime}}\left(\bar{\phi}_{Q} \psi_{\frac{4 N}{Q}}\left(\frac{Q}{*}\right)\right)=\overline{\psi_{Q^{\prime}}(Q)} f \mid \tilde{W}_{Q Q^{\prime}}(\psi)
$$

Proof. The proof proceeds along a standard line, but to check the automorphy factors, one must exercise a little care. First note that if $\chi=\left(\frac{Q}{*}\right)$, then the $Q$ and $4 N / Q$-parts

$$
\chi_{Q}=\left\{\begin{array}{ll}
\left(\frac{Q}{*}\right) & \text { if } Q \equiv 1(\bmod 4) \\
\left(\frac{-Q}{*}\right) & \text { if } Q \equiv 3(\bmod 4)
\end{array} \quad \chi_{4 N / Q}= \begin{cases}1 & \text { if } Q \equiv 1(\bmod 4) \\
\left(\frac{-1}{*}\right) & \text { if } Q \equiv 3(\bmod 4)\end{cases}\right.
$$

Then, as one considers the cases $Q \equiv \pm 1(\bmod 4)$, one must recall that

$$
\left(\frac{-1}{Q w}\right)^{1 / 2}=\left(\frac{-1}{Q}\right)^{1 / 2}\left(\frac{-1}{w}\right)^{1 / 2} \varepsilon(Q, w)
$$

where

$$
\varepsilon(Q, w)=\left\{\begin{aligned}
-1 & \text { if } Q \equiv w \equiv 3(\bmod 4) \\
1 & \text { otherwise }
\end{aligned}\right.
$$

Finally one uses the quadratic reciprocity law for Jacobi symbols.

Next we have the

Proposition 2.9. Let $f \in S_{k / 2}(4 N, \psi)$, with $Q$ as above, and let $p$ be a prime, $p \times N$. Then

$$
f\left|\tilde{T}_{k / 2,4 N, \psi}\left(p^{2}\right)\right| \tilde{W}_{Q}(\phi)=\phi_{Q}\left(p^{2}\right) f\left|\tilde{W}_{Q}(\phi)\right| \tilde{T}_{k / 2,4 N, \bar{\psi}_{Q} \psi_{\frac{\alpha Q}{Q}}\left(\frac{Q}{*}\right)}\left(p^{2}\right) .
$$

Proof. We sketch the basic idea. Let $\tilde{\tau}=\left\{\tau, p^{1 / 2}\right\} \in \mathscr{G}$, where $\tau=\left(\begin{array}{ll}1 & 0 \\ 0 & p^{2}\end{array}\right)$. The Hecke operator $\tilde{T}_{k / 2}\left(p^{2}\right)$ is described by the action of the double coset $p^{(k / 4)-1} \Delta_{0}(4 N) \tilde{\tau} \Delta_{0}(4 N)$. If we write

$$
\Delta_{0}(4 N) \tilde{\tau} \Delta_{0}(4 N)=\bigcup_{\nu} \Delta_{0}(4 N) \tilde{\tau}_{\nu},
$$

then we need to show that

$$
f\left|\tilde{T}_{k / 2,4 N, \psi}\left(p^{2}\right)\right| \tilde{W}_{Q}(\phi)=\sum_{\nu} \phi\left(a_{\nu}\right) f\left|\tilde{\tau}_{\nu}\right| \tilde{W}_{Q}(\phi)
$$




$$
\begin{aligned}
& =\phi_{Q}\left(p^{2}\right) \sum_{\nu^{\prime}} \bar{\phi}_{Q}\left(a_{\nu^{\prime}}\right) \phi_{\frac{4 N}{Q}}\left(a_{\nu^{\prime}}\right)\left(\frac{Q}{a_{\nu^{\prime}}}\right) f\left|\tilde{W}_{Q}(\phi)\right| \tilde{\tau}_{\nu^{\prime}} \\
& =\phi_{Q}\left(p^{2}\right) f\left|\tilde{W}_{Q}(\phi)\right| \tilde{T}_{k / 2,4 N, \bar{\psi}_{Q} \psi_{\frac{4 N}{Q}}\left(\frac{Q}{*}\right)}\left(p^{2}\right)
\end{aligned}
$$

where if $\tilde{\tau}=\left\{\tau_{\nu}, \varphi_{\nu}\right\} \in \mathscr{G}, a_{\nu}$ is the (1,1)-entry of $\tau_{\nu}$.

In fact, we show that for each $\nu$ there is a unique $\nu^{\prime}$ so that

$$
\phi\left(a_{\nu}\right) f\left|\tilde{\tau}_{\nu}\right| \tilde{W}_{Q}=\phi_{Q}\left(p^{2}\right) \bar{\phi}_{Q}\left(a_{\nu^{\prime}}\right) \phi_{\frac{4 N}{Q}}\left(a_{\nu^{\prime}}\right)\left(\frac{Q}{a_{\nu^{\prime}}}\right) f\left|\tilde{W}_{Q}\right| \tilde{\tau}_{\nu^{\prime}}
$$

Using Shimura's notation [8] for the expression of the double coset as a union of right cosets,

$$
\Delta_{0}(4 N) \tilde{\tau} \Delta_{0}(4 N)=\bigcup_{b=0}^{p^{2}-1} \Delta_{0}(4 N) \alpha_{b}^{*} \cup \bigcup_{h=0}^{p-1} \Delta_{0}(4 N) \beta_{h}^{*} \cup \Delta_{0}(4 N) \sigma^{*}
$$

we show that in the correspondence $\tilde{\tau}_{\nu} \leftrightarrow \tilde{\tau}_{\nu^{\prime}}$, we can relate $\alpha_{b}^{*} \leftrightarrow \alpha_{b^{\prime}}^{*}, \beta_{h}^{*} \leftrightarrow \beta_{h^{\prime}}^{*}$ and $\sigma^{*}$ with itself. Finally, since $p \nmid 4 N$, we may assume that in the definition of $\gamma_{Q}\left(W_{Q}\right)$, we have $z \equiv 0\left(\bmod p^{2}\right)$.

Next we describe the commutation relations of the shift operator and the $\tilde{W}_{Q^{-}}$operator.

Proposition 2.10. Let $M$ be a positive integer, and let $Q$ be an odd divisor of $M N$ with $\operatorname{gcd}(Q, 4 M N / Q)=1$. Then for $f \in S_{k / 2}(4 N, \phi)$ we have

$$
\begin{aligned}
f\left|B_{M}\right| \tilde{W}_{Q}^{A M N}\left(\phi\left(\frac{M}{*}\right)\right)= \\
\qquad \begin{array}{ll}
\bar{\phi}_{Q}(M) f\left|\tilde{W}_{Q}(\phi)\right| B_{M} & \text { if } \operatorname{gcd}(Q, M)=1 \\
\left(\frac{-1}{M}\right)^{k / 2} \bar{\phi}_{4 N M / Q}(M) M^{-k / 2} f \mid \tilde{W}_{Q / M}^{A N}(\phi) & \text { if } M \mid Q
\end{array}
\end{aligned}
$$

where the superscripts on the $\tilde{W}$-operators indicate the levels of the forms on which they operate.

Proof. This proof is analogous to the others, however it does require a case analysis based upon the congruence class of $M$ modulo 4 .

With these preliminary results in hand, we now address the interaction of the $\tilde{W}_{Q^{-}}$operator with oldforms and newforms. 
Proposition 2.11. Let $N$ be a positive integer, $Q$ an odd divisor of $N$ with $\operatorname{gcd}(Q, 4 N / Q)=1$, and $\phi$ an even Dirichlet character defined modulo $4 N$. Then $\tilde{W}_{Q}(\phi)$ takes $S_{k / 2}^{-}(4 N, \phi)$ to $S_{k / 2}^{-}\left(4 N, \bar{\phi}_{Q} \psi_{4 N / Q}\left(\frac{Q}{*}\right)\right)$, and in particular takes oldforms to oldforms.

Proof. It is clear from Proposition 2.9, that $\tilde{W}_{Q}$ takes Hecke eigenforms to Hecke eigenforms. Let $f \in S_{k / 2}^{-}(4 N, \phi)$. By linearity, it is enough to suppose that for some prime $q \mid N$ either $f \in S_{k / 2}(4 N / q, \phi)$ (provided $\phi$ is defined modulo $4 N / q)$, or $f=g \mid B_{q}$ for some $g \in S_{k / 2}\left(4 N / q, \phi\left(\frac{q}{*}\right)\right)\left(\right.$ provided $\left.\phi\left(\frac{q}{*}\right)\right)$ is defined modulo $4 N / q)$.

For the first case, write

$$
W_{Q}=W_{Q}^{4 N}=\gamma_{Q}^{4 N} \delta_{Q}=\left(\begin{array}{cc}
x & y \\
\frac{4 N}{Q} z & Q w
\end{array}\right) \delta_{Q} .
$$

We have two subcases depending upon whether $q \mid Q$ or not. Note that if $q \Varangle Q$, then $Q \mid 4 N / q$. We now write

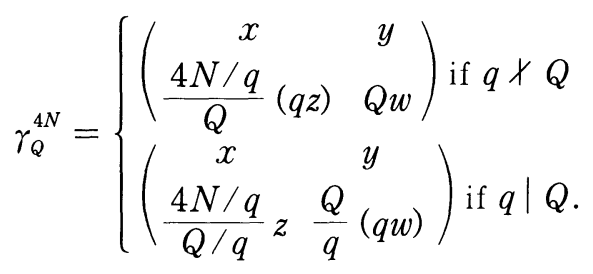

Notice that if $q \Varangle Q$, then $\gamma_{Q}^{4 N}$ is a valid representative for $\gamma_{Q}^{4 N / q}$ (needed to define $\tilde{W}_{Q / M}^{4 N / q}$ ), while if $q \mid Q$, then $\gamma_{Q}^{4 N}$ is a valid representative for $\gamma_{Q / q}^{4 N / q}$. Thus we have

$$
f \mid \tilde{W}_{Q}^{A N}(\phi)= \begin{cases}\kappa f \mid \tilde{W}_{Q}^{A N / q}(\phi) & \text { if } q \Varangle Q \\ \kappa^{\prime} f\left|\tilde{W}_{Q / q}^{4 N / q}\right| B_{q} & \text { if } q \mid Q\end{cases}
$$

for constants $\kappa$ and $\kappa^{\prime}$. In either (sub)case, $f \mid \tilde{W}_{Q}^{4 N}(\phi) \in S_{k / 2}^{-}\left(4 N, \bar{\psi}_{Q} \psi_{4 N / Q}\left(\frac{Q}{*}\right)\right)$.

In the case that $f=g \mid B_{q}$, we have by Proposition 2.10 that

$$
f\left|\tilde{W}_{Q}^{A N}(\phi)=g\right| B_{q} \mid \tilde{W}_{Q}^{4 N}(\phi)
$$




$$
= \begin{cases}\bar{\phi}_{Q}(q) g\left|\tilde{W}_{Q}^{A N / q}\left(\phi\left(\frac{q}{*}\right)\right)\right| B_{q} & \text { if } q \Varangle Q \\ \left(\frac{-1}{q}\right)^{k / 2} \bar{\phi}_{4 N q / Q}(q) q^{-k / 2} g \mid \tilde{W}_{Q / q}^{A N / q}\left(\phi\left(\frac{q}{*}\right)\right) & \text { if } q \mid Q,\end{cases}
$$

which again shows that $f \mid \tilde{W}_{Q}^{4 N}(\phi) \in S_{k / 2}^{-}\left(4 N, \bar{\phi}_{Q} \psi_{4 N / Q}\left(\frac{Q}{*}\right)\right)$ as required.

Our final proposition provides the last tools needed to complete the proof of Theorem 2.6.

Proposition 2.12. Let $N$ be a positive integer, $Q$ an odd divisor of $N$ with $(Q, 4 N / Q)=1$, and $\phi$ an even Dirichlet character defined modulo $4 N$.

1. For $f, g \in S_{k / 2}(4 N, \psi),\langle f, g\rangle=\left\langle f\left|\tilde{W}_{Q}, g\right| \tilde{W}_{Q}\right\rangle$.

2. The operator $\tilde{W}_{Q}(\phi)$ takes $S_{k / 2}^{+}(4 N, \phi)$ to $S_{k / 2}^{+}\left(4 N, \bar{\psi}_{Q} \psi_{4 N / Q}\left(\frac{Q}{*}\right)\right)$, and in particular takes newforms to newforms.

3. If $\phi$ is quadratic and $N$ odd and squarefree, then $S_{k / 2}^{+}(4 N, 1)$ and $S_{k / 2}^{+}(4 N, \phi)$ are isomorphic as modules for the Hecke algebra.

Proof. The first statement is obvious, since for all $\tilde{\rho} \in \mathscr{G},\langle f \mid \tilde{\rho}, g\rangle=\left\langle f, g \mid \tilde{\rho}^{-1}\right\rangle$. For the second statement, let $f \in S_{k / 2}^{+}(4 N, \psi)$ be a newform. By Proposition 2.9, $f \mid \tilde{W}_{Q}(\phi)$ is again a simultaneous Hecke eigenform. We need only to show that it lies in $S_{k / 2}^{+}\left(4 N, \bar{\psi}_{Q} \psi_{4 N / Q}\left(\frac{Q}{*}\right)\right)$. Let $g \in S_{k / 2}^{-}\left(4 N, \bar{\psi}_{Q} \psi_{4 N / Q}\left(\frac{Q}{*}\right)\right)$. Then by Proposition 2.11, $g \mid \tilde{W}_{Q}\left(\bar{\phi}_{Q} \psi_{4 N / Q}\left(\frac{Q}{*}\right)\right) \in S_{k / 2}^{-}(4 N, \phi)$, and $g\left|\tilde{W}_{Q}\left(\bar{\phi}_{Q} \psi_{4 N / Q}\left(\frac{Q}{*}\right)\right)\right| \tilde{W}_{Q}(\phi)=$ $\kappa g$ with $|\kappa|=1$. Thus

$$
\begin{aligned}
\left\langle f \mid \tilde{W}_{Q}(\phi), g\right\rangle & =\kappa\left\langle f\left|\bar{W}_{Q}(\phi), g\right| \tilde{W}_{Q}\left(\bar{\phi}_{Q} \psi_{4 N / Q}\left(\frac{Q}{*}\right)\right) \mid \tilde{W}_{Q}(\phi)\right\rangle \\
& =\kappa\left\langle f, g \mid \bar{W}_{Q}\left(\bar{\phi}_{Q} \psi_{4 N / Q}\left(\frac{Q}{*}\right)\right)\right\rangle=0
\end{aligned}
$$

as required. For the third statement, since $N$ is odd and squarefree, we may take $\phi=\left(\frac{Q}{*}\right)$ for some divisor $Q$ of $N$ (necessarily satisfying $(Q, 4 N / Q)=1$ ). Then $\tilde{W}_{Q}(1)$ maps $S_{k / 2}^{+}(4 N, 1)$ to $S_{k / 2}^{+}(4 N, \phi)$. Moreover, by Proposition $2.9, f$ and $f \mid \tilde{W}_{Q}(1)$ have the same Hecke eigenvalues, and since $\tilde{W}_{Q}(1)$ is an "involution", the proof is complete. 


\section{Decomposition theorems-Prime power level}

In this section, we decompose the distinguished subspace $S^{\emptyset}\left(4 q^{m}, \phi\right)$ defined by Ueda [12] in terms of the space of newforms as defined in this paper. We give a decomposition of both the full space of cusp forms $S_{k / 2}^{ø}\left(4 q^{m}, \psi\right)$ and of the Kohnen subspace $S_{k / 2}^{\emptyset}\left(4 q^{m}, \psi\right)_{K}$ where $q$ is an odd prime, $m \geq 2$, and $\psi$ an even quadratic Dirichlet character defined modulo $4 q^{m}$. We begin with Ueda's definition of $S^{\emptyset}$ using twisting operators. We remind the reader of Ueda's notation [12].

Write $N=4 q^{m}=M_{1} M_{2}^{+}$with $M_{1}=1$ and $M_{2}^{+}=q^{m}$. Let $\Pi=\{q\}$. Write $S\left(4 q^{m}, \phi\right)$ to represent either $S_{k / 2}\left(4 q^{m}, \phi\right)$ or $S_{k / 2}\left(4 q^{m}, \phi\right)_{K}$. While we have defined the subspace of oldforms $S_{k / 2}^{-}\left(4 q^{m}, \phi\right)$ in equation (2.1), we need to make sure the same definition makes sense in the Kohnen subspace. That is, we want to make the definition

$$
\begin{aligned}
& S_{k / 2}^{-}(4 N, \phi)_{K}=\sum_{\substack{q|N \\
\operatorname{cond}(\psi)| 4 N / q}} S_{k / 2}(4 N / q, \phi)_{K} \\
& +\sum_{\substack{q\left|N \\
\operatorname{cond}\left(\psi\left(\frac{q}{*}\right)\right)\right| 4 N / q}} S_{k / 2}\left(4 N / q, \phi\left(\frac{q}{*}\right)\right)_{K} \mid B_{q} .
\end{aligned}
$$

We need only to check that $S_{k / 2}\left(4 N / q, \phi\left(\frac{q}{*}\right)\right)_{K} \mid B_{q} \subset S_{k / 2}(4 N, \phi)_{K}$. Clearly there is no issue with the level and character. The only condition to check is that the constraints on the Fourier coefficients which define the Kohnen subspace are met.

Let $g=\sum a(n) x^{n} \in S_{k / 2}\left(4 N / q, \phi\left(\frac{q}{*}\right)\right)_{K}$, and write $g \mid B_{q}=\sum b(n) x^{n} \in$ $S_{k / 2}(4 N, \phi)$. Since $\phi$ is quadratic, we may write $\phi=\left(\frac{N_{0}}{*}\right)$ for some positive divisor $N_{0}$ of $N$. Let $\varepsilon=\left(\frac{-1}{N_{0}}\right)$. Then $g \mid B_{q} \in S_{k / 2}(4 N, \phi)_{K}$ provided $b(n)=0$ for $\varepsilon(-1)^{\frac{k-1}{2}} n \equiv 2,3(\bmod 4)$.

Since $g \in S_{k / 2}\left(4 N / q, \phi\left(\frac{q}{*}\right)\right)_{K}$, and $\phi\left(\frac{q}{*}\right)=\left(\frac{q N_{0}}{*}\right)$, we know that $a(n)$ $=0$ if $\left(\frac{-1}{q}\right) \varepsilon(-1) \frac{k-1}{2} n \equiv 2,3(\bmod 4)$. Now $b(n)=a(n / q)=0$ unless $q \mid n$, so write $n=q n_{0}$. Then $b(n)=a\left(n_{0}\right)$. Since $\left(\frac{-1}{q}\right) q \equiv 1(\bmod 4)$, if $\varepsilon(-1)^{\frac{k-1}{2}} n$ $\equiv 2,3(\bmod 4)$, then 


$$
\varepsilon(-1)^{\frac{k-1}{2}} n \equiv \varepsilon(-1)^{\frac{k-1}{2}} n_{0} q \equiv\left(\frac{-1}{q}\right) \varepsilon(-1)^{\frac{k-1}{2}} n_{0} \equiv 2,3(\bmod 4)
$$

so that $a\left(n_{0}\right)=b(n)=0$ as required.

We define the subspace of oldforms in the Kohnen subspace by equation (3.1), and define the subspace of newforms $S_{k / 2}^{+}(4 N, \psi)_{K}$ as the orthogonal complement of $S_{k / 2}^{-}(4 N, \psi)_{K}$ in $S_{k / 2}(4 N, \psi)_{K}$. Note that these subspaces are different than the ones Ueda defines, but it is precisely the point of this section to compare these subspaces by decomposing one in terms of the other.

So with $S\left(4 q^{m}, \phi\right)$ representing either the full space of cusp forms or the Kohnen subspace, we let $S^{ \pm}\left(4 q^{m}, \phi\right)$ represent the corresponding subspaces of old or newforms as defined in and immediately following equations (2.1) and (3.1).

We begin our comparison of the decomposition of $S\left(4 q^{m}, \psi\right)$ using Ueda's twisting operators and our notion of newforms. It follows from Propositions 1.5, 1.10 , and 1.11 of [12] that for $m \geq 2$,

$$
S\left(4 q^{m}, \phi\right)=S^{\emptyset}\left(4 q^{m}, \phi\right) \perp S\left(4 q^{m-1}, \phi\left(\frac{q}{*}\right)\right) \mid B_{q}
$$

where $\perp$ denotes orthogonal sum. Moreover, by definition, we know that

$$
S\left(4 q^{m}, \phi\right)=S^{+}\left(4 q^{m}, \phi\right) \perp S^{-}\left(4 q^{m}, \phi\right),
$$

and from the definitions, $S\left(4 q^{m-1}, \phi\left(\frac{q}{*}\right)\right) \mid B_{q} \subset S^{-}\left(4 q^{m}, \phi\right)$. Thus $S^{+}\left(4 q^{m}, \phi\right)$ $\subset S^{\emptyset}\left(4 q^{m}, \phi\right)$.

Now by definition,

$$
S^{-}\left(4 q^{m}, \phi\right)=S\left(4 q^{m-1}, \phi\right)+S\left(4 q^{m-1}, \phi\left(\frac{q}{*}\right)\right) \mid B_{q}
$$

We compute that

$$
S\left(4 q^{m-1}, \phi\right) \cap S\left(4 q^{m-1}, \phi\left(\frac{q}{*}\right)\right)\left|B_{q}=S\left(4 q^{m-2}, \phi\left(\frac{q}{*}\right)\right)\right| B_{q}
$$

as follows. First it is obvious that the RHS is contained in the LHS. Now let $f=$ $g \mid B_{q} \in S\left(4 q^{m-1}, \psi\right)$ with $g \in S\left(4 q^{m-1}, \psi\left(\frac{q}{*}\right)\right)$. Since $f$ has level $4 n^{m-1}$, by Propositions 1.10 and 1.11 of [12], we have that $g \in S\left(4 q^{m-2}, \phi\left(\frac{q}{*}\right)\right)$ or $g=0$, which is what we required.

It follows that 


$$
S^{-}\left(4 q^{m}, \psi\right)=S\left(4 q^{m-1}, \phi\left(\frac{q}{*}\right)\right) \mid B_{q} \oplus\left[\left(S\left(4 q^{m-2}, \phi\left(\frac{q}{*}\right)\right) \mid B_{q}\right)^{\perp} \text { in } S\left(4 q^{m-1}, \psi\right)\right] .
$$

On the other hand, just as in (3.2) for $m \geq 3$,

$$
S\left(4 q^{m-1}, \psi\right)=S^{\emptyset}\left(4 q^{m-1}, \psi\right) \perp S\left(4 q^{m-2}, \phi\left(\frac{q}{*}\right)\right) \mid B_{q},
$$

hence

$$
S^{-}\left(4 q^{m}, \psi\right)=S^{\emptyset}\left(4 q^{m-1}, \psi\right) \oplus S\left(4 q^{m-1}, \psi\left(\frac{q}{*}\right)\right) \mid B_{q} .
$$

Thus for $m \geq 3$ we have

$$
\begin{aligned}
S\left(4 q^{m}, \phi\right) & =S^{\emptyset}\left(4 q^{m}, \phi\right) \perp S\left(4 q^{m-1}, \phi\left(\frac{q}{*}\right)\right) \mid B_{q} \\
& =S^{+}\left(4 q^{m}, \phi\right) \perp\left[S^{\emptyset}\left(4 q^{m-1}, \phi\right) \oplus S\left(4 q^{m-1}, \phi\left(\frac{q}{*}\right)\right) \mid B_{q}\right] .
\end{aligned}
$$

If only the last sum in the above expression were an orthogonal sum, then by the uniqueness of orthogonal complements, we could easily deduce the decomposition:

$$
S^{\emptyset}\left(4 q^{m}, \psi\right)=S^{+}\left(4 q^{m}, \phi\right) \perp S^{\emptyset}\left(4 q^{m-1}, \phi\right) .
$$

However, the sum is not necessarily orthogonal. Nonetheless, we will eventually deduce exactly this identity with $\perp$ replaced by $\oplus$. Unfortunately, the proof is a bit more indirect.

We begin by establishing the above identity as an isomorphism as modules for the Hecke algebra $H(q)$ generated by all Hecke operators $\tilde{T}_{k / 2}\left(p^{2}\right)$ with $(p, 2 q)=1$.

Theorem 3.1. Let $q$ be an odd prime, $m \geq 2$ an integer, and $\psi$ an even quadratic Dirichlet character defined modulo $4 q^{m}$. Then as $H(q)$-submodules of $S\left(4 q^{m}, \phi\right)$,

$$
S^{\emptyset}\left(4 q^{m}, \phi\right) \cong \begin{cases}S^{+}\left(4 q^{m}, \phi\right) \oplus S^{\emptyset}\left(4 q^{m-1}, \phi\right) & \text { if } m \geq 3 \text { or } m=2, \phi=1 \\ S^{+}\left(4 q^{2}, \phi\right) \oplus S^{+}(4 q, \phi) & \text { if } m=2 \text { and } \phi=\left(\frac{q}{*}\right)\end{cases}
$$

Proof. We must separate the cases $m \geq 3$ and $m=2$. First consider $m \geq 3$. Recall that $H(q)$ is a commutative semisimple algebra. Since $\phi$ is quadratic, by Lemma 5 of [9] $\tilde{T}_{k / 2}\left(p^{2}\right)=\tilde{T}_{k / 2,4 q^{m}, \phi}\left(p^{2}\right)$ is hermitian, and by easy computations 
(see [12]), we can verify that all of the summands in equation (3.4) are $H(q)$ submodules of $S\left(4 q^{m}, \psi\right)$. Thus the equalities in equation (3.4) also hold as isomorphisms as $H(q)$-modules:

$$
\begin{aligned}
S\left(4 q^{m}, \psi\right) & \cong S^{\emptyset}\left(4 q^{m}, \phi\right) \oplus S\left(4 q^{m-1}, \phi\left(\frac{q}{*}\right)\right) \mid B_{q} \\
& \cong S^{+}\left(4 q^{m}, \phi\right) \oplus S^{\emptyset}\left(4 q^{m-1}, \phi\right) \oplus S\left(4 q^{m-1}, \phi\left(\frac{q}{*}\right)\right) \mid B_{q}
\end{aligned}
$$

Using the semisimplicity of $H(q)$ and canceling like terms yields the result in this case. For $m=2$, equation (3.3) is not valid. By the conventions in Ueda [12], $S(4 q, \psi)=S^{\emptyset}(4 q, \psi)$ and $S\left(4, \phi\left(\frac{q}{*}\right)\right) \mid B_{q}$ is not necessarily zero.

As above, we do have that

$$
\begin{aligned}
S\left(4 q^{2}, \phi\right) & =S^{\emptyset}\left(4 q^{2}, \phi\right) \oplus S\left(4 q, \phi\left(\frac{q}{*}\right)\right) \mid B_{q} \\
& =S^{+}\left(4 q^{2}, \phi\right) \oplus S\left(4 q, \phi\left(\frac{q}{*}\right)\right) \mid B_{q} \oplus\left[\left(S\left(4, \phi\left(\frac{q}{*}\right)\right) \mid B_{q}\right)^{\perp} \text { in } S(4 q, \phi)\right] .
\end{aligned}
$$

Moreover, these equalities also hold as $H(q)$-module isomorphisms.

If $\phi=1$, then $S\left(4, \phi\left(\frac{q}{*}\right)\right)=0$, so equation (3.5) and semisimplicity of $H(q)$ yield

$$
S^{\emptyset}\left(4 q^{2}, \phi\right) \cong S^{+}\left(4 q^{2}, \psi\right) \oplus S(4 q, \psi)=S^{+}\left(4 q^{2}, \phi\right) \oplus S^{\emptyset}(4 q, \phi)
$$

where $S^{\emptyset}(4 q, \psi)=S(4 q, \phi)$ by Ueda's conventions.

If $\phi \neq 1\left(\right.$ and hence $\left.\phi=\left(\frac{q}{*}\right)\right)$, we need to compute $\left[\left(S(4,1) \mid B_{q}\right)^{\perp}\right.$ in $\left.S\left(4 q,\left(\frac{q}{*}\right)\right)\right]$. But

$$
\begin{aligned}
& \quad S\left(4 q,\left(\frac{q}{*}\right)\right)=S^{+}\left(4 q,\left(\frac{q}{*}\right)\right) \perp S^{-}\left(4 q,\left(\frac{q}{*}\right)\right)=S^{+}\left(4 q, \psi\left(\frac{q}{*}\right)\right) \perp S(4,1) \mid B_{q}, \\
& \text { so }\left[\left(S(4,1) \mid B_{q}\right)^{\perp} \text { in } S\left(4 q,\left(\frac{q}{*}\right)\right)\right]=S^{+}\left(4 q,\left(\frac{q}{*}\right)\right) .
\end{aligned}
$$

Thus

$$
S\left(4 q^{2},\left(\frac{q}{*}\right)\right) \cong S^{ø}\left(4 q^{2},\left(\frac{q}{*}\right)\right) \oplus S(4 q, 1) \mid B_{q}
$$




$$
\cong S^{+}\left(4 q^{2},\left(\frac{q}{*}\right)\right) \oplus S(4 q, 1) \mid B_{q} \oplus S^{+}\left(4 q,\left(\frac{q}{*}\right)\right)
$$

which yields $S^{\emptyset}\left(4 q^{2},\left(\frac{q}{*}\right)\right) \cong S^{+}\left(4 q^{2},\left(\frac{q}{*}\right)\right) \oplus S^{+}\left(4 q,\left(\frac{q}{*}\right)\right)$ as required.

As a corollary we have

COROLlary 3.2. Let the notation be as above, and put $\mu=\operatorname{ord}_{q}(\operatorname{cond}(\phi))(=0$ or 1), then

$$
S^{\emptyset}\left(4 q^{m}, \psi\right) \cong \bigoplus_{l=\mu}^{m} S^{+}\left(4 q^{l}, \psi\right)
$$

Proof. It is immediate from Theorem 3.1 that

$$
S^{\emptyset}\left(4 q^{m}, \phi\right) \cong \begin{cases}\bigoplus_{l=2}^{m} S^{+}\left(4 q^{l}, \psi\right) \oplus S^{\emptyset}(4 q, \psi) & \text { if } \phi=1 \\ \bigoplus_{l=2}^{m} S^{+}\left(4 q^{l}, \psi\right) \oplus S^{+}(4 q, \psi) & \text { if } \phi=\left(\frac{q}{*}\right) .\end{cases}
$$

If $\phi=\left(\frac{q}{*}\right)$, we are done. To go further, we recall Ueda's convention [12] that $S^{\emptyset}(4 q, \phi)=S(4 q, \phi)$. If $\psi=1$, then $S^{-}(4 q, 1)=S(4,1)=S^{+}(4,1)$, so $S(4 q, 1)$ $=S^{+}(4 q, 1) \oplus S^{-}(4 q, 1)=S^{+}(4 q, 1) \oplus S^{+}(4,1)$, as required.

Remark 3.3. Multiplicities of newforms. When $S\left(4 q^{m}, \phi\right)=S_{k / 2}\left(4 q^{m}, \phi\right)_{K}$, Ueda [12] has established a newform theory and strong multiplicity-one theorem. First, he decomposes the space $S^{\emptyset}$ using twisting operators. In our case, this decomposition is quite simple: $S^{\emptyset}=S^{\emptyset,+} \oplus S^{\emptyset,-}$, where $S^{\emptyset, \pm}=\left\{f \in S^{\emptyset}: f \mid R_{q}= \pm f\right\}$ and $R_{q}$ is the twisting operator associated to $\left(\frac{*}{q}\right)$. He then decomposes each subspace $S^{\emptyset, \pm}$ into a direct sum of a space of oldforms $\tilde{\mathfrak{\Im}}^{\emptyset, \pm}$ and its orthogonal complement $\mathfrak{S}^{\emptyset, \pm}$ (newforms). In our case $S^{\emptyset, \pm}=\mathfrak{S}^{\emptyset, \pm}$. By Theorem 3.11 of [12], each summand $\mathfrak{S}^{\emptyset, \pm}$ satisfies a strong multiplicity-one theorem. In particular, since

$$
S^{\emptyset}=S^{\emptyset,+} \oplus S^{\emptyset,-}=\Im^{\emptyset,+} \oplus \Im^{\emptyset,-} \cong \bigoplus_{l=\mu}^{m} S^{+}\left(4 q^{l}, \phi\right),
$$

we must have that newforms in the spaces $S^{+}\left(4 q^{l}, \phi\right)$ occur with multiplicity one or two. This is analogous to our Theorem 2.3 which states a more precise result for the full space of cusp forms having squarefree level. 
We now show that $S^{\emptyset}\left(4 q^{m-1}, \phi\right) \subset S^{\emptyset}\left(4 q^{m}, \phi\right)$ (for $m \geq 3$ ). To do so, we first need to compute the adjoint of the operator $U_{q}$. The proposition below is stated for the full space of cusp forms. The action on the Kohnen subspace is the same, as we discuss following the proof.

Proposition 3.4. Let $q$ be an odd prime and $N$ a positive integer. Suppose that $q \mid N$, and $\phi$ is an even Dirichlet character defined modulo $4 N$. Let $U_{q}^{*}: S_{k / 2}\left(4 N, \phi\left(\frac{q}{*}\right)\right) \rightarrow S_{k / 2}(4 N, \phi)$ be the adjoint of the $U_{q}$ operator. Then for $g \in S_{k / 2}\left(4 N, \phi\left(\frac{q}{*}\right)\right)$,

$$
g\left|U_{q}^{*}=q^{k / 2} g\right| B_{q} \mid \operatorname{Tr}_{4 N}^{4 N q}(\phi)
$$

where $B_{q}$ is the shift operator and $\operatorname{Tr}$ is the trace operator.

Proof. Let $\Gamma_{1}=\Gamma_{1}(4 N), \Delta_{1}=\Delta_{1}(4 N), \alpha=\left(\begin{array}{ll}1 & 0 \\ 0 & q\end{array}\right)$ and $\xi=\left[\alpha, q^{1 / 4}\right] \in \mathscr{G}$. Then $U_{q}=\Delta_{1} \xi \Delta_{1}$. By Lemma 1.9 of [11] (using Proposition 1.1 and equation (1.14) of [8]), we have $U_{q}^{*}=\Delta_{1} \xi^{\prime} \Delta_{1}$, where $\xi^{\prime}=\left[\alpha^{\prime}, q^{1 / 4}\right] \in \mathscr{G}, \alpha^{\prime}=\left(\begin{array}{ll}q & 0 \\ 0 & 1\end{array}\right)$.

If $\alpha_{\mu}=\left(\begin{array}{cc}1 & 0 \\ 4 N \mu & 1\end{array}\right)$, then since $q \mid N$, we have

$$
\Gamma_{1}=\Gamma_{1}(4 N)=\cup_{\mu=1}^{q} \Gamma_{1}(4 N q) \alpha_{\mu} \quad \text { and } \quad \Gamma_{1} \alpha \Gamma_{1}=\cup_{\mu=1}^{q} \Gamma_{1} \alpha \alpha_{\mu} .
$$

Again by Proposition 1.1 and equation (1.14) of Shimura [8]

$$
\Delta_{1}(4 N) \xi^{\prime} \Delta_{1}(4 N)=\cup_{\mu=1}^{q} \Delta_{1}(4 N) \xi^{\prime} \alpha_{\mu}^{*}
$$

where $\alpha_{\mu}^{*}=\left[\alpha_{\mu}, j\left(\alpha_{\mu}, \tau\right)\right] \in \Delta_{1} \subset \mathscr{G}$.

So for $g \in S_{k / 2}\left(4 N, \phi\left(\frac{q}{*}\right)\right)$, we have

$$
\begin{aligned}
g \mid U_{q}^{*} & =g\left|\Delta_{1} \xi^{\prime} \Delta_{1}=\operatorname{det}\left(\alpha^{\prime}\right)^{k / 4-1} \sum_{\mu=1}^{q} g\right| \xi^{\prime} \alpha_{\mu}^{*} \\
& =q^{k / 2-1} \sum_{\mu=1}^{q} g\left|B_{q}\right| \alpha_{\mu}^{*}=q^{k / 2-1} \sum_{\mu=1}^{q} \bar{\psi}\left(d_{\mu}\right)\left(g \mid B_{q}\right) \mid \alpha_{\mu}^{*}
\end{aligned}
$$

where $d_{\mu}=1$ is the $d$-entry of $\alpha_{\mu}$

$$
=q^{k / 2-1}\left[\Gamma_{1}(4 N): \Gamma_{1}(4 N q)\right] g\left|B_{q}\right| \operatorname{Tr}_{4 N}^{4 N q}(\phi)=q^{k / 2} g\left|B_{q}\right| \operatorname{Tr}_{4 N}^{4 N q}(\phi)
$$

as required. 
We note that the same expression makes sense as well for the Kohnen subspace, provided $N$ is odd so the space is defined. Apriori, the adjoint of the $U_{q}$ operator restricted to the Kohnen subspace is the orthogonal projection of the adjoint $U_{q}^{*}$ into the Kohnen subspace, but we have already observed that the $B_{q}$ operator preserves the Kohnen subspace, and since the trace operator is the adjoint of the inclusion map, it does as well.

Proposition 3.5. Let $m \geq 2$, and $f \in S^{\emptyset}=S^{\emptyset}\left(4 q^{m}, \phi\right)$. Then $f \mid U_{q}=0$.

Proof. Recall that $S^{\emptyset}=S^{\emptyset,+} \oplus S^{\emptyset,-}$ where $S^{\emptyset, \pm}=\left\{f \in S^{\emptyset}: f \mid R_{q}= \pm f\right\}$, and $R_{q}$ is the twisting operator associated to $\left(\frac{*}{q}\right)$. Clearly, it is enough to verify the result for $f \in S^{\emptyset, \pm}$. For such an $f=\sum a_{n} x^{n}, f \mid R_{q}= \pm f$ means that $a_{n}=0$ whenever $q \mid n$. Thus $f \mid U_{q}=\sum a_{n q} x^{n}=0$.

Proposition 3.6 For $m \geq 3, S^{\emptyset}\left(4 q^{m-1}, \phi\right) \subset S^{\emptyset}\left(4 q^{m}, \phi\right)$.

Proof. Let $f \in S^{\emptyset}\left(4 q^{m-1}, \phi\right) \subset S\left(4 q^{m}, \phi\right)$. Then

$$
\begin{aligned}
f \in S^{\emptyset}\left(4 q^{m}, \psi\right) & \Leftrightarrow\left\langle f, g \mid B_{q}\right\rangle_{4 q^{m}, \psi}=0 \text { for all } g \in S\left(4 q^{m-1}, \phi\left(\frac{q}{*}\right)\right) \\
& \Leftrightarrow\left\langle f, g\left|B_{q}\right| \operatorname{Tr}_{4 q^{m-1}}^{4 q^{m}}\right\rangle_{4 q^{m-1}, \psi}=0 \text { for all } g \in S\left(4 q^{m-1}, \phi\left(\frac{q}{*}\right)\right) \\
& \Leftrightarrow\left\langle f, g \mid U_{q}^{*}\right\rangle=0 \text { for all } g \\
& \Leftrightarrow\left\langle f \mid U_{q}, g\right\rangle=0 \text { for all } g
\end{aligned}
$$

which is true by the previous proposition.

Corollary 3.7. For $m \geq 3, S^{\emptyset}\left(4 q^{m}, \psi\right)=S_{k / 2}^{+}\left(4 q^{m}, \phi\right) \oplus S^{\emptyset}\left(4 q^{m-1}, \psi\right)$.

Proof. We have already verified that both summands are contained in the RHS. Moreover, $S_{k / 2}^{+}\left(4 q^{m}, \phi\right) \cap S^{\emptyset}\left(4 q^{m-1}, \phi\right)=0$ since $S^{\emptyset}\left(4 q^{m-1}, \phi\right) \subset S\left(4 q^{m-1}, \phi\right)$ $\subset S_{k / 2}^{-}\left(4 q^{m}, \phi\right)$. Thus the RHS is a summand of the LHS. But by Theorem 3.1, both sides are isomorphic as $H(q)$-modules, and hence as finite dimensional vector spaces from which the assertion follows.

\section{REFERENCES}

[1] W. Kohnen, Newforms of half-integral weight, J. reine angew. Math., 333 (1982), 
$32-72$.

[2] W. Li, Newforms and functional equations, Math. Annalen, 212 (1975), 285-315.

[3] M. Manickam, B. Ramakrishnan, T. Vasudevan, On the theory of newforms of half-integral weight, J. Number Theory, 34 (1990), 210-224.

[4] S. Niwa, On Shimura's trace formula, Nagoya Math J., 66 (1977),183-202.

[ 5 ] J.-P.Serre and H. Stark, Modular forms of weight $1 / 2$, In Lecture Notes in Math. 627, Springer-Verlag, Berlin and New York (1977), 27-67.

[6] T. Shemanske, Cuspidal newforms and chracter twists, J. reine angew. Math., 328 (1981), 59-71.

[7] T. Shemanske and L. Walling, Determining multiplicities of half-integral weight newforms, Pacific J. Math., 167 (1995), 345-383.

[ 8 ] G. Shimura, On modular forms of half-integral weight, Annals of Math., 97 (1973), $440-481$.

[9] G. Shimura, The critical values of certain zeta functions associated with modular forms of half-integral weight, J. Math. Soc. Japan, 33 (1981), 649-672.

[10] M. Ueda, The decomposition of the spaces of cusp forms of half-integral weight and the trace formula of Hecke operators, J. Math. Kyoto U., 28 (1988), 505-555.

[11] M. Ueda, The trace formula of twisting operators on the spaces of cusp forms of half-integral weight and some trace relations, Japanese J. Math., 17 (1991), $83-135$.

[12] M. Ueda, On twisting operators and newforms of half-integral weight, Nagoya Math J., 131 (1993), 135-205.

Department of Mathematics

6188 Bradley Hall

Dartmouth College, Hanover,

New Hampshire 03755-3551, U.S.A.

E-mail address:Thomas.Shemanske@dartmouth.edu 
